\title{
Estudo comparativo da porcentagem das áreas cortical, trabecular e do canal medular do fêmur de ratos hipertensos (SHR) e normotensos Comparative study of cortical, trabecular and marrow areas percentage in hypertensive (SHR) and normotensive rats femur
}

\author{
Andresa Costa PEREIRA \\ Doutoranda do Programa de Pós-Graduação em Biopatologia Bucal - Faculdade de Odontologia de São José dos \\ Campos - Universidade Estadual Paulista - UNESP - São José dos Campos - SP - Brasil
}

\section{Susana Ungaro AMADEI}

Doutoranda do Programa de Pós-Graduação em Biopatologia Bucal - Faculdade de Odontologia de São José dos Campos - Universidade Estadual Paulista - UNESP - São José dos Campos - SP - Brasil

\section{Vanessa Ávila Sarmento SILVEIRA}

Doutoranda do Programa de Pós-Graduação em Biopatologia Bucal - Faculdade de Odontologia de São José dos Campos - Universidade Estadual Paulista - UNESP - São José dos Campos - SP - Brasil

\section{Ivan BALDUCCI}

Professor do Departamento de Odontologia Social e Clínica Infantil - Faculdade de Odontologia de São José dos Campos - Universidade Estadual Paulista - UNESP - São José dos Campos - SP - Brasil

\section{Horácio FAIG-LEITE}

Professor Adjunto do Departamento de Biociências e Diagnostico Bucal - Faculdade de Odontologia de São José dos Campos - Universidade Estadual Paulista - UNESP - São José dos Campos - SP - Brasil

\begin{abstract}
Resumo
Os portadores da hipertensão arterial sistêmica apresentam uma tendência à perda óssea devido às alterações no metabolismo do cálcio. O objetivo deste trabalho foi avaliar e comparar as porcentagens das áreas cortical, trabecular e do canal medular em ratos hipertensos (SHR) e normotensos. Foram utilizados 24 ratos, sendo 12 SHR (6 machos e 6 fêmeas) e 12 normotensos (6 machos e 6 fêmeas). Após o sacrifício e dissecção do fêmur direito, foi realizado um corte transversal (aproximadamente $1 \mathrm{~mm}$ de espessura) na região de maior diâmetro do osso e o material imerso em hipoclorito de sódio $2 \%$ para retirada do tecido mole. As peças foram fotografadas em um estereomicroscópio e as imagens submetidas ao programa Image-J® para mensuração $\left(\mathrm{em} \mathrm{mm}^{2}\right)$ das áreas cortical, trabecular e do canal medular. Os valores encontrados foram transformados em porcentagens e submetidos à ANOVA e ao teste de Tukey $(5 \%)$. Verificouse que o valor de porcentagem do canal medular foi maior nos ratos hipertensos, tanto machos $(\mathrm{p}=0,02)$ quanto fêmeas $(p=0,01)$. Em relação às médias das porcentagens das áreas cortical e trabecular, os animais hipertensos apresentaram valores menores, embora não estatisticamente significativos. Também não foram encontradas diferenças significativas entre machos e fêmeas. Concluiu-se que os animais hipertensos machos e fêmeas apresentaram aumento da porcentagem da área do canal medular, provavelmente em consequiência à redução dos ossos cortical e trabecular, o que sugere um aumento na reabsorção óssea nos hipertensos.
\end{abstract}

\section{UNITERMOS}

Hipertensão; tecido ósseo, morfologia. 


\section{INTRODUÇÃo}

A hipertensão arterial sistêmica tem sido relacionada com a osteoporose em humanos ${ }^{9}$ e animais hipertensos ${ }^{1,13}$. O mecanismo desta relação ainda não está claro, porém, é sugerido que ocorra devido às mudanças no metabolismo do cálcio presente nos hipertensos ${ }^{1,3,4,6,8}$.

Na literatura, há relatos de uma redução no conteúdo de cálcio ósseo ${ }^{1}$ e na densidade mineral óssea nos ratos espontaneamente hipertensos (SHR) $)^{1,3,7}$.

A redução da massa óssea em hipertensos também foi evidenciada em alguns estudos, sendo justificada por um desequilíbrio na remodelação deste tecido, com um predomínio do processo de reabsorção óssea ${ }^{1,2,12}$.

Sabe-se que as características gerais da hipertensão primária podem ser alteradas por influência dos hormônios sexuais ${ }^{10,11}$. Entretanto, a maioria dos estudos experimentais que avaliam as características ósseas dos SHR foi realizada apenas em machos, sendo que poucos comparam as diferenças entre os $\operatorname{sexos}^{3,13}$.

Dessa maneira, o objetivo deste trabalho foi avaliar as porcentagens correspondentes às áreas cortical, trabecular e canal medular em fêmures de ratos hipertensos (SHR) comparando-os com normotensos e verificando as diferenças existentes entre machos e fêmeas.

\section{Material e Método}

Foram utilizados 24 ratos, com aproximadamente 22 semanas de idade, sendo 12 SHR (6 machos e 6 fêmeas) e 12 controles normotensos (6 machos e 6 fêmeas). Após o sacrifício, os fêmures direitos dos animais foram removidos, dissecados e cortados transversalmente no local de maior diâmetro da região proximal da diáfise. Os cortes (aproximadamente $1 \mathrm{~mm}$ de espessura) foram colocados em hipoclorito de sódio $2 \%$ durante dez horas, para retirada do tecido mole, e posteriormente foram analisados em estereomicroscópio com aumento de $12.5 \mathrm{x}$. As peças foram fotografadas e as imagens analisadas, com auxílio do

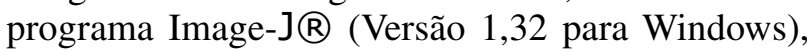
de domínio público (http://rsb.info.nih.gov/ij/download.html), do Instituto Nacional de Saúde (NIH), Bethesda, EUA.

A área total (A) e as áreas correspondentes ao osso cortical (AC), ao trabecular (AT) e ao canal medular (AM) foram circundadas e mensuradas em $\mathrm{mm}^{2}$ (Figura 1). Os valores foram transformados em porcentagem: porcentagem cortical $(\mathrm{PC}=\mathrm{AC} / \mathrm{A})$, porcentagem trabecular ( $\mathrm{PT}=\mathrm{AT} / \mathrm{A})$ e porcentagem do canal medular (PM=AM/A) e avaliados separadamente. Os dados foram apresentados como média e desvio-padrão (média $\pm \mathrm{DP}$ ).

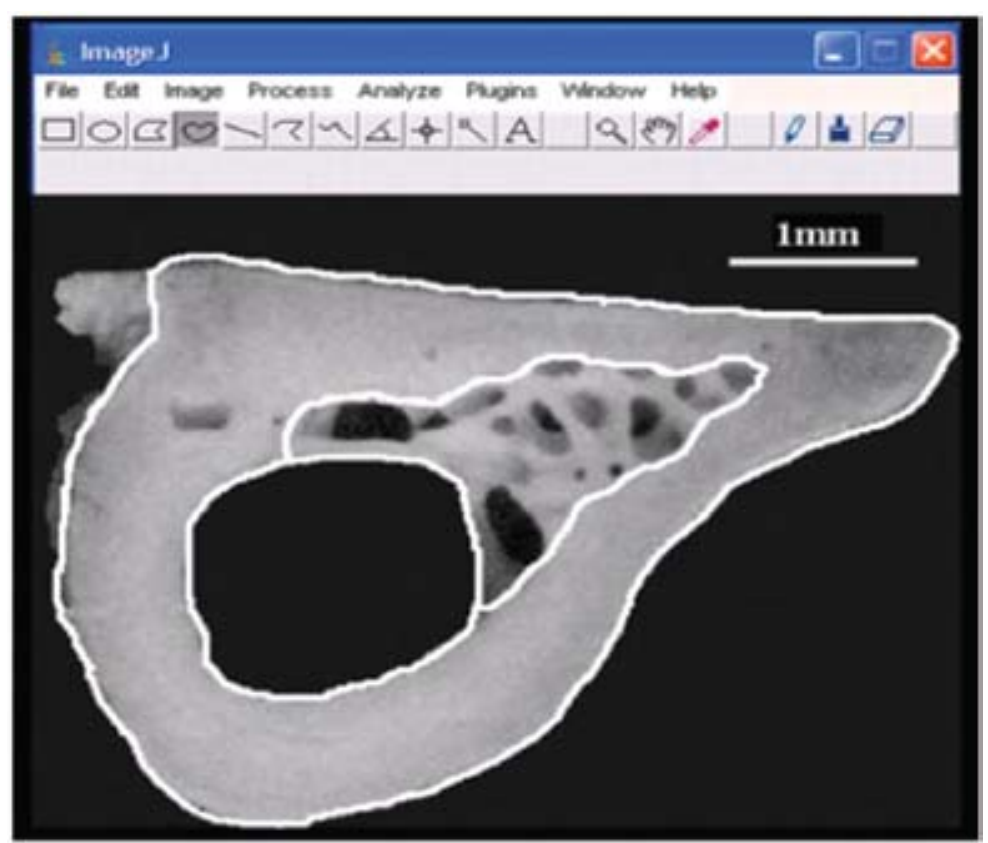

FIGURA 1 - Metodologia empregada para a morfometria das áreas ósseas cortical, trabecular e do canal medular (em mm2), realizada por meio do programa Image $\mathbf{J}$. 
Para cada porcentagem (PC, PT ou PM), a análise estatística foi realizada em duas etapas. Na primeira, houve a realização da análise de variância (ANOVA-dois fatores) e do teste de Tukey (5\%) para todos os grupos de animais, avaliando a relação entre a presença da hipertensão e o sexo. Na segunda, foi aplicado ANOVA (um fator) para machos e fêmeas independentemente.

\section{Resultados}

As médias das porcentagens das áreas cortical, trabecular e do canal medular estão expostas na Tabela 1 e representadas na Figura 2.

Tabela 1 - Médias \pm desvio-padrão correspondentes às porcentagens das áreas cortical (PC), trabecular (PT) e do canal medular (PM) nos animais normotensos e hipertensos, machos e fêmeas

\begin{tabular}{c|c|c|c}
\hline & PC & PT & PM \\
\hline Normotenso Macho & $60,4 \pm 5$ & $17,93 \pm 4,99$ & $21,66 \pm 2,17$ \\
Hipertenso Macho & $58,31 \pm 6,38$ & $12,84 \pm 7,76$ & $28,86 \pm 3,96$ \\
Normotenso Fêmea & $59,05 \pm 5,5$ & $17,74 \pm 8,37$ & $23,21 \pm 4,93$ \\
Hipertenso Fêmea & $58,07 \pm 5,47$ & $10,23 \pm 2,17$ & $31,37 \pm 4,01$ \\
\hline
\end{tabular}

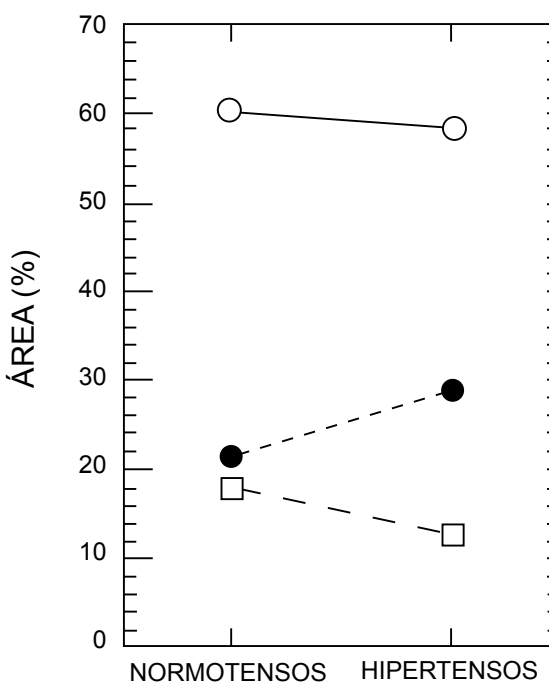

MACHOS

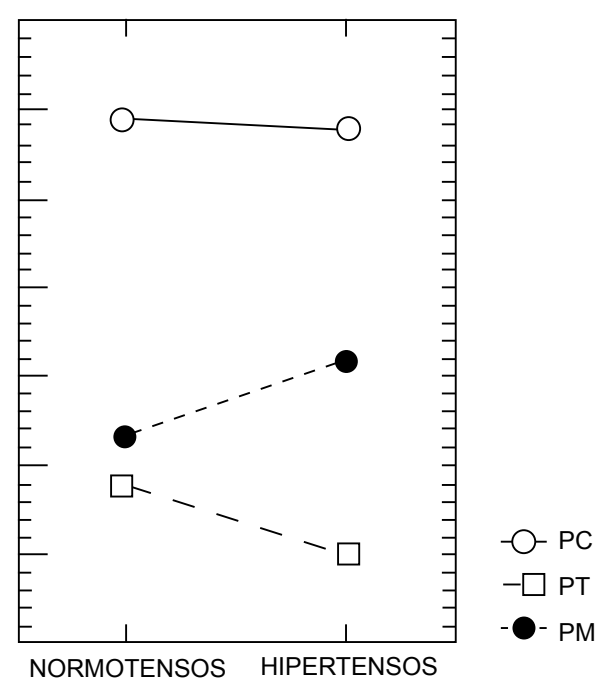

FÊMEAS

FIGURA 2 - Médias dos dados referentes às porcentagens das áreas cortical (PC), trabecular (PT) e do canal medular (PM) nos animais normotensos e hipertensos, machos e fêmeas. 
Após a aplicação do ANOVA (dois fatores) para PC, PT e PM, verificou-se que o p referente à interação entre o sexo e a presença da hipertensão não foi estatisticamente significante para nenhuma das porcentagens.

Como machos e fêmeas apresentaram padrões semelhantes (Figura 2), aplicamos ANOVA (um fator) para os valores de PC, PT e PM dos animais machos e fêmeas separadamente. Pudemos verificar $p$ significante apenas para os valores de $\mathrm{PM}$, tanto para machos $(\mathrm{p}=0,02)$ quanto para fêmeas $(\mathrm{p}=0,01)$, com a presença de maior canal medular nos animais hipertensos de ambos os grupos. Os valores de p para PC e PT foram, respectivamente: $\mathrm{p}=0,54$ e 0,20 (machos) e $\mathrm{p}=0,76$ e 0,59 (fêmeas).

Na Figura 3, pode-se notar os cortes ósseos referentes aos animais normotensos e hipertensos, machos e fêmeas.
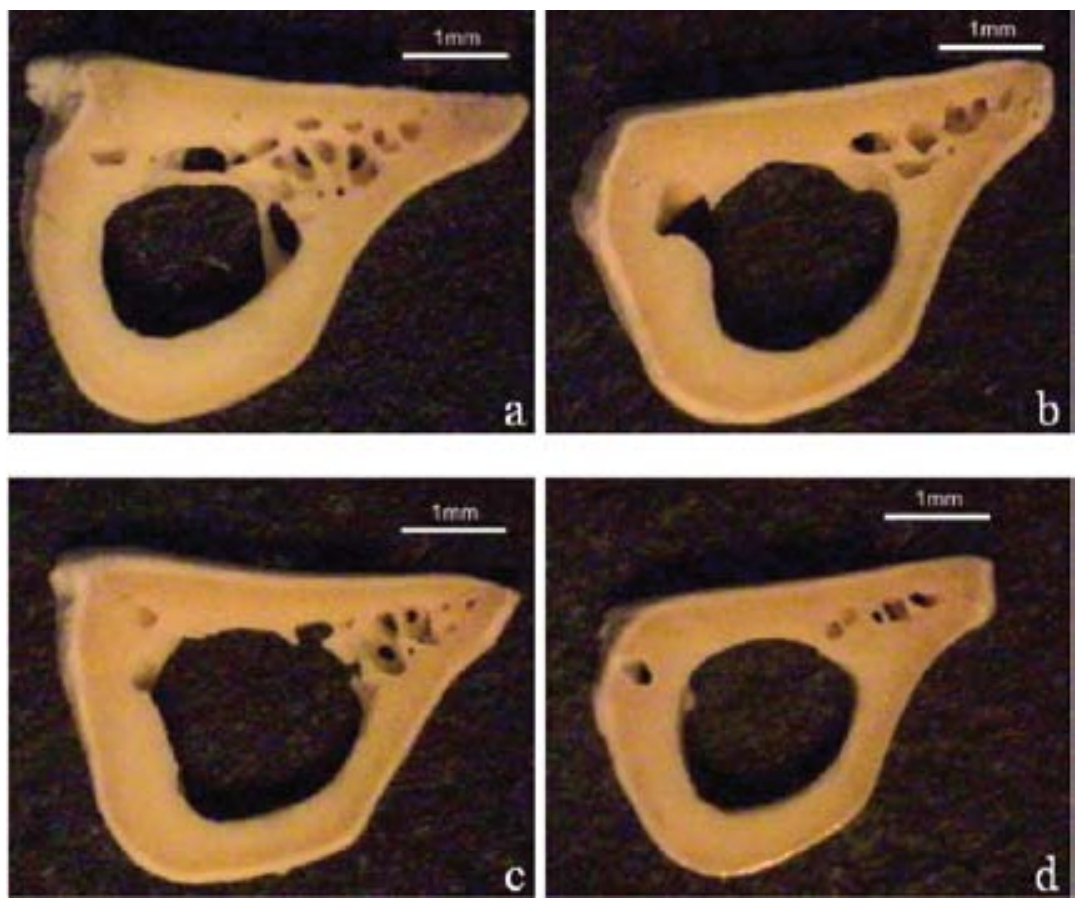

FIGURA 3 - Fotografia das peças ósseas em estereomicroscópio (aumento original de 12.5). a) animal do grupo normotenso macho, b) animal do grupo normotenso fêmea, c) animal do grupo hipertenso macho, d) animal do grupo hipertenso fêmea.

\section{Discussão}

O presente estudo demonstra uma alteração no valor percentual da área do canal medular dos fêmures dos animais hipertensos, fato este que se relaciona com achados de diversos autores ${ }^{1,3,4,7,12,13}$, os quais notaram características ósseas distintas nos SHR, quando comparados aos controles normotensos.

No nosso trabalho, os valores de PC, PT e PM foram calculados em cortes transversais. Dessa forma, em relação aos valores corticais e medulares, usamos a mesma metodologia de outros estudos ${ }^{4,5,12}$, entretanto, para a mensuração dos valores trabeculares, a literatura relata a realização de cortes longitudinais ${ }^{12}$, o que difere do nosso trabalho.
Outra diferença é que os trabalhos encontrados na literatura avaliaram a morfometria óssea em tíbias $^{4,5,12}$, entretanto em nossa metodologia utilizamos fêmures.

Nossos valores referentes à PM foram estatisticamente maiores nos animais hipertensos em relação aos normotensos, tanto em machos quanto em fêmeas. Comparando com os trabalhos que avaliaram ratos machos, nossos achados confirmam os resultados de Izawa et al. ${ }^{4}$ (1985), porém discordam de Wang et al. ${ }^{12}$ (1993), os quais não encontraram nenhuma diferença estatística entre estes valores. Em relação ao canal medular das fêmeas, nosso trabalho difere do de Liang et al. ${ }^{5}$ (1997), pois estes autores não encontraram diferença estatística entre a PM das ratas hipertensas e normotensas. 
Apesar de não significantes, as médias referentes à $\mathrm{PC}$ e PT foram menores nos animais hipertensos de ambos os sexos e dessa maneira, acreditamos que a discreta redução do osso cortical somada a diminuição do osso trabecular foi responsável pelo aumento do canal medular. O cálculo das áreas feito em porcentagem foi realizado justamente para não haver resultados distorcidos devido às diferentes áreas totais dos ossos, características que podem ser visualizadas na Figura 3.

Acredita-se que o mecanismo de redução da massa óssea nos ratos hipertensos ocorra devido à taxa de reabsorção exceder a formação óssea, com a atividade osteoclástica sendo dominante sobre a atividade osteoblástica $^{1,2,12}$. Wang et al. ${ }^{12}$ (1993) verificaram um aumento do número de osteoclastos e redução do número de osteoblastos e células osteoprogenitoras nos SHR, fato este, que pode justificar um aumento na reabsorção óssea dos ossos cortical e trabecular nos animais hipertensos, com conseqüente aumento do canal medular.

Em concordância com Wang et al. ${ }^{12}$ (1993) e Liang et al. ${ }^{5}$ (1997), não verificamos uma redução significativa da porcentagem de osso cortical para machos e fêmeas, respectivamente. Entretanto, é relatado que as diferenças entre o osso trabecular dos SHR e normotensos são muito maiores do que em relação ao osso cortical ${ }^{5}$.

Wang et al. ${ }^{12}$ (1993) verificaram uma redução significante do volume trabecular nos SHR e mostraram uma diminuição da espessura e do número de trabéculas, além de um aumento na separação trabecular em comparação com os normotensos. Em nossos resultados não verificamos diferenças estatísticas da porcentagem trabecular, apesar de uma discreta redução nos hipertensos, fato que pode ser justificado pela diferença na metodologia empregada.

Apesar da maioria dos estudos ter sido realizada em ratos machos, é importante a avaliação do compor- tamento ósseo em fêmeas, pois a osteoporose é muito mais prevalente em mulheres do que em homens ${ }^{5}$. O estudo de Liang et al. ${ }^{5}$ (1997) foi o único a avaliar a real influência hormonal nas ratas fêmeas hipertensas, pois estes autores compararam fêmeas ovariectomizadas e não ovariectomizadas. Nossos resultados mostraram valores semelhantes entre machos e fêmeas, já que as fêmeas por nós utilizadas apresentavam condições hormonais normais. Talvez se estas fêmeas fossem ovariectomizadas, encontraríamos valores diferenciados, uma vez que Liang et al. ${ }^{5}$ (1997) verificaram que a perda óssea foi maior nas ratas com deficiência de estrógeno, o que ressalta a importância clínica para mulheres pós-menopausa.

Os estudos comparando machos e fêmeas são raros. Em relação à taxa de reabsorção óssea, Wright $\& \operatorname{DeMoss}^{13}(2000)$ verificaram que na $24^{\text {a }}$ semana de vida dos animais, período semelhante ao do nosso experimento, houve uma clara diferença entre os sexos, com significante aumento na taxa de reabsorção nas fêmeas, principalmente SHR.

Em nosso estudo, não verificamos diferenças entre machos e fêmeas, concordando com Inoue et $\mathrm{al}^{3}{ }^{3}$ (1995), os quais não verificaram alteração no volume ósseo nem na espessura trabecular de machos e fêmeas.

\section{Conclusão}

Dessa maneira, os achados do nosso trabalho permitem concluir que os animais hipertensos apresentaram aumento da porcentagem da área do canal medular consequiente à redução dos ossos cortical e trabecular, o que nos leva a supor um aumento na taxa de reabsorção óssea nos hipertensos, relacionada talvez ao aumento do número de osteoclastos ${ }^{12} \mathrm{e}$ ao metabolismo alterado do cálcio ${ }^{1,4,6}$. 


\section{Abstract}

The systemic arterial hypertension patients present a bone loss tendency due to calcium metabolism alteration. The aim of this study was to evaluate and compare cortical, trabecular and marrow areas percentage in hypertensive (SHR) and normotensive rats. Twenty four rats were divided into 2 groups: 12 SHR (6 males and 6 females) and 12 normotenses (6 males and 6 females). After the sacrifice, the right femurs were dissected and a $1 \mathrm{~mm}$ transversal cut was performed in the largest bone region and the material was immersed into sodium hypochlorite $2 \%$ to soft tissue removal. The sections were photographed and analyzed by Image-J® program to measure cortical, trabecular and marrow areas (in $\left.\mathrm{mm}^{2}\right)$. The values were transformed in percentage and submitted to ANOVA and Tukey test (5\%). It was verified that the marrow area percentage was higher in hypertensive rats, in males $(p=0,02)$ and also females $(p=0,01)$. Although cortical and trabecular areas percentage were lower in hypertensive animals, these values were not significant. In addition, no difference was observed between males and females rats. In conclusion, males and females hypertensive animals presented a marrow area percentage increase maybe in consequence to cortical and trabecular area reduction, what suggests an increased bone resorption in hypertensive animals.

\section{UNITERMS}

hypertension; bone and bone; morphology.

\section{REFERÊNCIAS}

1. Barbagallo M, Quaini F, Baroni MC, Barbagallo CM, Boiardi L, Passeri $\mathrm{G}$ et al. Histological evidence of increased turnover in bone from spontaneously hypertensive rats. Cardioscience 1991 mar.; 2(1): $15-7$.

2. DeMoss DL, Wright GL. Sex and strain differences in whole skeletal development in the rat. Calcif Tissue Int 1998 feb.; 62(2): 153-7.

3. Inoue T, Moriya A, Goto K, Tanaka T, Inazu M. What is the difference of bone growth in SHR and SD rats? Clin Exp Pharmacol Physiol Suppl 1995 dec.; 22(1): 242-3.

4. Izawa Y, Sagara K, Kadota T, Makita T. Bone disorders in spontaneously hypertensive rat. Calcif Tissue Int 1985 dec.; 37(6): 605-7.

5. Liang H, Ma Y, Pun S, Stimpel M, Jee WS. Aging- and ovariectomyrelated skeletal changes in spontaneously hypertensive rats. Anat Rec 1997 oct.; 249(2): 173-80.

6. McCarron DA, Yung NN, Ugoretz BA, Krutzik S. Disturbances of calcium metabolism in the spontaneously hypertensive rat. Hypertension 1981 may/jun.; 3(3): 162-7.

7. Metz JA, Karanja N, Young EW, Morris CD, McCarron DA. Bone mineral density in spontaneous hypertension: differential effects of dietary calcium and sodium. Am J Med Sci 1990 oct.; 300(4): 225-30.

8. Oshima T, Young EW. Systemic and cellular calcium metabolism and hypertension. Semin Nephrol 1995 nov.; 15(6): 496-503.
9. Perez-Castrillon JL, Justo I, Silva J, Sanz A, Igea R, Escudero P et al. Bone mass and bone modeling markers in hypertensive postmenopausal women. J Hum Hypertens 2003 feb.; 17(2): 107-10.

10. Reckelhoff JF. Gender differences in the regulation of blood pressure Hypertension 2001 may; 37(5): 1199-208.

11. Reckelhoff JF, Zhang H, Srivastava K. Gender differences in development of hypertension in spontaneously hypertensive rats: role of the renin-angiotensin system. Hypertension 2000 jan.; 35(1): 480-3.

12. Wang TM, Hsu JF, Jee WS, Matthews JL. Evidence for reduced cancellous bone mass in the spontaneously hypertensive rat. Bone Miner 1993 mar.; 20(3): 251-64.

13. Wright GL, DeMoss D. Evidence for dramatically increased bone turnover in spontaneously hypertensive rats. Metabolism 2000 sep.; 49(9): 1130-3.

Recebido em: 12/10/06 Aprovado em: 05/06/07

Andresa Costa Pereira Departamento de Biociências e Diagnostico Bucal e-mail: andresa-cp@uol.com.br Av. Engenheiro Francisco José Longo, 777 - Jardim São Dimas 12245-000 - São José dos Campos - SP - Brasil Fone: (12) 39479037 - Fax: (12) 39479010 